\title{
Interaction of color and geometric cues in depth perception: When does "red" mean "near"?
}

Received: 13 February 2003 / Accepted: 27 November 2003/Published online: 10 February 2004

(C) Springer-Verlag 2004

\begin{abstract}
Luminance and color are strong and self-sufficient cues to pictorial depth in visual scenes and images. The present study investigates the conditions under which luminance or color either strengthens or overrides geometric depth cues. We investigated how luminance contrast associated with the color red and color contrast interact with relative height in the visual field, partial occlusion, and interposition to determine the probability that a given figure presented in a pair is perceived as "nearer" than the other. Latencies of "near" responses were analyzed to test for effects of attentional selection. Figures in a pair were supported by luminance contrast (Experiment 1) or isoluminant color contrast (Experiment 2) and combined with one of the three geometric cues. The results of Experiment 1 show that the luminance contrast of a color (here red), when it does not interact with other colors, produces the same effects as achromatic luminance contrasts. The probability of "near" increases with the luminance contrast of the color stimulus, the latencies for "near" responses decrease with increasing luminance contrast. Partial occlusion is found to be a strong enough pictorial cue to support a weaker red luminance contrast. Interposition cues lose out against cues of spatial position and partial occlusion. The results of Experiment 2, with isoluminant displays of varying color contrast, reveal that red color contrast on a light background supported by any of the three geometric cues wins over green or white supported by any of the three geometric cues. On a dark background, red color contrast supported by the interposition cue loses out against green or white color contrast supported by partial occlusion. These findings reveal that color is not an independent depth cue, but is
\end{abstract}

C. R. C. Guibal $(\bowtie)$

Department of Optometry and Visual Science, City University

London, Northampton Square, London, EC 1V 0HB, UK

E-mail: c.r.c.guibal@city.ac.uk

B. Dresp

CNRS and Faculté des Sciences du Sport,

Université Montpellier 1, 700 avenue du Pic Saint-Loup,

34090 Montpellier, France strongly influenced by luminance contrast and stimulus geometry. Systematically shorter response latencies for stronger "near" percepts demonstrate that selective visual attention reliably detects the most likely depth cue combination in a given configuration.

\section{Introduction}

The relative visibility of objects, their color, their relative size or relative position within the visual field, the local interposition of their contours, and the partial occlusion of their shapes represent attributes that provide critical cues for processing figure-ground relationships in human visual perception. Such cues, frequently referred to as pictorial depth cues, determine how we perceive what appears nearer to us or further away in a two-dimensional (2-D) display.

The role of luminance contrast in depth perception

The relative visibility of an object represented in a picture or a scene is mostly determined by its luminance contrast. The fact that relative visibility correlates with pictorial depth was established by findings from original studies on visual perception under water and on land showing that luminance contrast simulates the laws of aerial perspective (Ross, 1967). These observations were confirmed later by Egusa (1983) and Rohaly and Wilson (1991), who showed that the perceived depth of a given region within the visual field is, indeed, determined by the brightness of that region. O'Shea, Blackburn, and Ono (1994) demonstrated, under conditions of both binocular and monocular viewing, that the higher-contrast stimulus of a pair systematically appears nearer than the lower-contrast stimulus.

Moreover, when the luminance cue was combined with a size cue, which is supposed to mimic linear perspective where stimuli with a larger size appear generally 
nearer than stimuli with a smaller size, it was shown that the size cue loses out against the luminance cue. "Near" judgments in response to stimuli of different sizes combined with weaker luminance contrasts never exceeded the chance level whereas "near" responses to different sizes combined with stronger luminance contrasts increased with size up to values well above the chance level (O'Shea et al., 1994). Recently, Dresp, Durand, and Grossberg (2002) have shown, with achromatic stimuli, that it is luminance contrast that determines which shape of a pair of shapes will be seen as nearer when a strong luminance cue is put into competition with pictorial cues of interposition and partial occlusion. The apparent hierarchy of effects, showing that partial occlusion wins over interposition at weaker luminance contrasts and, under certain conditions, takes considerably shorter exposure durations to resolve, suggests cooperative and competitive interactions that depend on which cue is available in combination with another one in a given configuration.

Whether colored stimuli produce identical effects remains to be clarified. Color is widely assumed to have an independent status as a cue to depth perception because of the intrinsically competitive nature of short-wavelength colors and long-wavelength colors identified in the well-known phenomenon of color stereopsis.

\section{The contribution of color stereopsis}

The color of shapes or objects is another powerful, selfsufficient, and presumably independent cue to depth in the visual field. The phenomenon of color stereopsis refers to the effect of color contrast on the perceived depth of stimuli when they are viewed binocularly, through a convex lens or with the unaided eye, and when their luminance contrast does not vary. Experiments in color stereopsis have shown that long-wavelength stimuli, such as red or yellow, compete with short-wavelength stimuli such as blue or green, when participants are requested to judge which color appears closer (Brewster, 1851). When viewed binocularly, with the aided or unaided eye (Hartridge, 1947; Dengler \& Nitschke, 1993), reds and yellows appear closer than blues or greens. Whether this intrinsically competitive nature of color cues to depth, predicted by the wavelength of the stimuli, is under the influence of other cues such as luminance contrast or stimulus geometry remains to be clarified. Dengler and Nitschke (1993) have shown that changes in contrast at the borders of isoluminant orange and blue lines or squares may induce depth reversals. When the colors were lighter than the background, the long-wavelength color orange was seen in front of the short-wavelength color blue; when the colors were darker than the background, blue was seen in front. While these findings clearly indicate that color cues interact with luminance contrast, their significance with regard to pictorial depth perception has not been further investigated.
Cue cooperation or cue competition?

Finding out about cue interactions, or how cues may cooperate or compete in a given configuration, is critical to our understanding of how depth percepts are extracted from a complex image or visual scene. Earlier theories (e.g., Gibson, 1950) have emphasized the importance of cooperative interactions, or cue combinations, claiming that information provided by multiple cues would have to be combined to generate unified percepts of shapes and their relative depth within the visual field. More recent models (e.g., Landy, Maloney, \& Young, 1995) consider both cooperative and competitive interactions between different depth cues. Cue combination, or cooperation of cues, would occur when two qualitatively different depth cues contribute to the depth percept at a given location. Conflict, or competition between cues, would occur in situations where an unambiguous cue fails to strengthen an ambiguous one. Knill (2003) proposed a mixture model based on cue cooperation. A mechanism that computes a conditional probability function, which is the product of the likelihood functions for each given cue, predicts when a single cue will have enough weight to effectively determine the resulting percept. In this mixture model, based on cooperative weights, cue competition is, like in Grossberg's (1997) FACADE theory, the inevitable consequence of cue selection. Although all cues available in a given configuration cooperate in the first instance, cues with little relative weight may then be overridden by a cue with stronger relative weight. It can be assumed that probabilistic mechanisms of this kind drive many perceptual processes, including attention. The extensive literature on visual search, for example, demonstrates that those combinations of stimulus attributes that are the most likely to be relevant in a given situation promote selective visual attention.

\section{Luminance contrast, color, and attentional selection}

Some objects automatically attract attention away from other objects in the visual field. Objects with a stronger luminance contrast, for example, may benefit from selective visual attention when presented together with objects of a lesser contrast (e.g., Yantis \& Jones, 1991; Dresp \& Grossberg, 1999). Similarly, some colors may attract attention away from other colors in the visual field, the color red in particular. It has been known for some time that red is readily seen as nearer than other colors (e.g., Verhoeff, 1928). This not only holds true for the controlled conditions of experiments in color stereopsis, but is also experienced in complex visual scenes where factors other than color stereopsis contribute to the emergence of depth percepts. The red signal in traffic lights is one such example (Bugalski, 1967). To demonstrate the link between spatial attention, color, and depth perception, Nakayama and Silverman (1986) have shown that the visual detection of targets defined by 
conjunctions of color and depth in a visual search task is faster than the detection of targets defined by a single attribute. The implications of a correlation between perceived depth in geometric configurations and selective visual attention are discussed further by Nakayama, Shimojo, and Ramachandran (1990).

If such a correlation exists, the probability that a given object is seen to be nearer than another object in the visual field should correlate with the response latencies of near percepts. In particular, under normal viewing conditions where luminance contrast, color contrast, stimulus geometry, and visual attention interact, the cue combinations that yield the most likely depth percept should promote selective visual attention. Then, a strong luminance or color cue combined with a strong geometric cue should produce shorter response latencies than a weaker luminance or color cue combined with a weaker geometric cue. Measuring response latencies to probe attentional mechanisms has proved successful in visual search experiments (e.g., Nakayama \& Silverman, 1986), showing that certain combinations of visual cues produce significantly shorter response latencies than others in a given task context. Such effects are commonly explained in terms of facilitation or inhibition of attentional selection (e.g., Goolsby \& Suzuki, 2001).

Cooperative and competitive interactions between luminance contrast and geometric cues to pictorial depth

Dresp, Durand, and Grossberg (2002) investigated the effect of interactions between luminance contrast and geometric cues, such as partial surface occlusion and contour interposition, on the probability that a given figure of a pair is seen as "nearer" than the other. They found that strong achromatic luminance contrasts, although they cooperate with any given geometrical cue they define at weaker intensities, generate competitive interactions with geometric cues to depth, especially with interposition cues. Partial occlusion was found to "survive" the competition against a strong luminance cue significantly better than an interposition cue, although both interposition and partial occlusion clearly resolved ambiguities about which stimulus lies in front of each other in the pictorial plane. The fact that partial occlusion cues win against interposition cues in the competition with a strong luminance cue was explained by the relative boundary advantage produced by the partial occlusion cue compared with the interposition cue. In configurations with a partial occlusion cue, a larger amount of visible boundary information is provided by the figure lying in front (see Fig. 1a for an illustration). Grossberg's FACADE model (1997) predicts that this relative boundary advantage contributes to a stronger depth percept because it generates comparatively stronger signals in the Boundary Contour System (BCS) of the model, which is orientation selective and detects boundaries. The BCS interacts with the Feature Contour System (FCS) of the model, which a
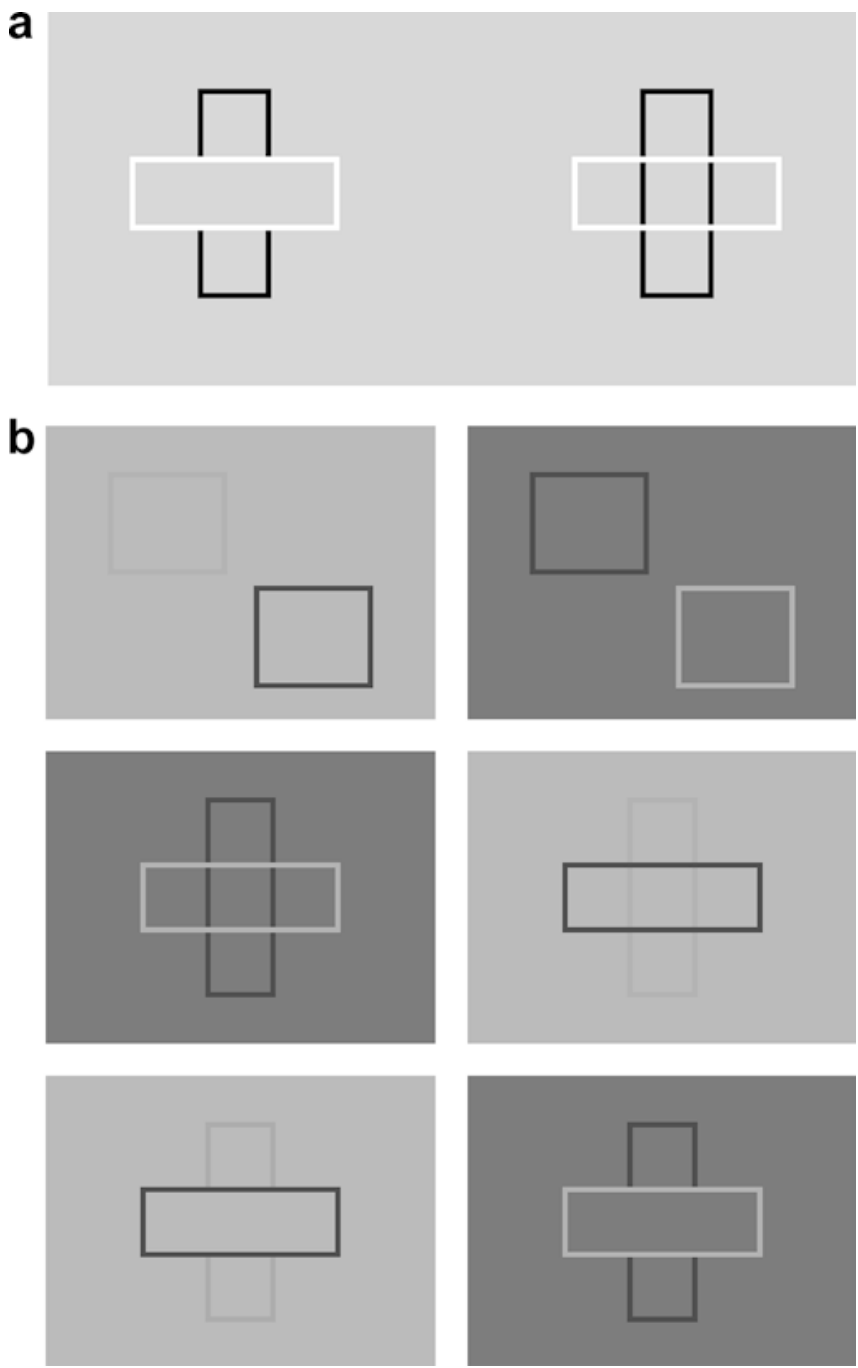

Fig. 1 a In the cross configurations shown here, geometric cues of partial surface occlusion (left) and contour interposition (right) cause the horizontal rectangle to be seen as "nearer" to the observer than the vertical rectangle when no other cue to pictorial depth competes with the geometric cues. The partial occlusion cue generates a local boundary advantage. Grossberg's FACADE model (Grossberg, 1997) explains how the local boundary advantage can make the partial occlusion cue win over the interposition cue in the competition with other depth cues such as luminance contrast in achromatic stimuli (Dresp et al., 2002). b The stimuli presented in Experiment 1 consisted of pairs of red squares or rectangles. To vary the relative visibility of the figures of a given pair, the luminance of the background was varied. In the condition with the positional cue, two spatially separated squares were presented (top). The positional cue systematically favored the right square of a given pair. Conditions with interposition cue (middle), or with cues of partial occlusion (bottom), systematically favored the horizontal rectangles in the cross configurations

detects luminance contrasts within image regions defined by boundaries. The combined monocular outputs of BCS and FCS cooperatively feed into a selective BCSFCS mechanism that groups input from a given location in the visual field. The output of this BCS-FCS interaction determines whether a stimulus in a given location "survives" against stimuli in other locations. Stimuli "surviving" this monocular, competitive stage of the 
FACADE model are selected and integrated into a binocular form representation (Grossberg, 1997).

Confirming some key hypotheses of the FACADE theory, Dresp et al. (2002) showed that:

1. A strong enough contrast cue can "override" a geometric cue

2. The relative strength of a geometric cue can be assessed by its capacity to compete with another strong depth cue such as achromatic luminance contrast

3. Cortical mechanisms sensitive to contrast intensity and selective to stimulus geometry, such as those suggested by the FACADE model, provide a plausible account of these interactions

The present experiments were designed to extend Dresp et al.'s (2002) study to colored stimuli. The aim of the first experiment was to determine whether dark and bright luminance contrasts of a particular color generate effects of relative visibility similar to those found with dark and bright achromatic contrasts. Competitive interactions between a strong luminance cue of a given color and geometric cues were investigated. The FACADE hypothesis tested in Dresp et al.'s (2002) study predicts a clear advantage of partial occlusion over interposition in the competition against a strong luminance cue because of the relative boundary advantage in stimulus pairs with the partial occlusion cue. In the present study, we added a cue of spatial position. The importance of spatial position as a pictorial depth cue was first mentioned by Leonardo da Vinci in his Trattato della Pittura (1651). Later, Rock, Shallo, and Schwartz (1978) and Bruno and Cutting (1988) showed that stimuli that are located at a lower position in the visual field are perceived as nearer than stimuli located higher in the visual field. This cue to depth is now referred to as the Relative-Height-In-TheVisual-Field cue, and a particularly suitable probe for the contribution of selective visual attention to depth perception. The geometric cue available in a pair of stimuli of different heights in the visual field is spatial location. In the case of the relative height cue, the perceptual system would readily expect objects presented at lower locations in the visual field to be nearer to the human eye because other geometric constraints need not be taken into account. The FACADE theory predicts that expectation driven by cues of spatial location acts as a powerful director of visual attention (Grossberg, 1997). Like the partial occlusion cue, the positional cue does not lead to a relative boundary advantage in a stimulus pair. At the same time, a cue of spatial location should attract attention to the most likely depth solution faster than an interposition cue given that, in the case of relative height, an image interpretation taking into account further geometric constraints is not necessary.

The aim of the second experiment was to clarify the status of color contrast as a depth cue with regard to geometrical cues such as partial occlusion, interposition, and relative height in the visual field. If color is, as widely supposed, a strong enough and largely independent cue to pictorial depth, no particular kind of interaction with geometrical cues would be expected. To test for effects of background luminance previously observed in color stereopsis, we presented isoluminant color pairs on dark and light backgrounds. Finally, to look for a possible correlation between selective visual attention and perceived depth, suggested by findings on visual search for targets defined by color and depth (e.g., Nakayama \& Silverman, 1986), we analyzed response times for "near" responses in the two experiments.

\section{Experiment 1: Luminance contrast of red versus partial occlusion, interposition, and relative height in the visual field}

We investigated the effects of luminance contrast and contrast polarity (dark contrasts versus bright contrasts) of red stimuli with constant chromaticity on the probability that a given figure of a pair is perceived as "nearer" than the other. The contrast polarity of the red stimuli was varied to test for possible functional asymmetries between bright and dark in the perception of "near" in colored figures (e.g., Dresp \& Fischer, 2001) with varying luminance contrast. Previously, Dresp et al. (2002) have shown that "near" percepts in pairs of achromatic figures were not affected by whether a dark figure overlapped a bright one, or a bright figure overlapped a dark one. This result ruled out functional asymmetries between dark and bright achromatic stimuli (e.g., Magnussen \& Glad, 1975), which may have influenced the perception of apparent depth.

Interactions between the red stimuli and geometric cues of partial surface occlusion, contour interposition, and relative height in the visual field of a given figure in a pair were tested. The experiment was designed so that the position cue systematically favored the stimulus displayed on the right-hand side at a lower position within the visual field compared with the left-hand side stimulus (see Fig. 1b, top). Interposition cues and cues of partial occlusion systematically favored the horizontal rectangle in the test configurations (see Fig. 1b, middle and bottom). Such a bias would produce a probability of 1 that squares on the right and horizontal rectangles are perceived as "nearer" if relative height, interposition, and partial occlusion were independent depth cues with equivalent strength, and if relative color visibility had no effect.

\section{Method}

\section{Participants}

Six women and five men participated in Experiment 1. They were all young volunteers with normal or corrected-to-normal vision and naive with regard to the purpose of the study. 
Stimuli

The stimuli were presented binocularly on a high-resolution computer screen (Sony, $60 \mathrm{~Hz}$, non-interlaced). They were generated with an IBM compatible PC (HP 486) equipped with a VGA Trident graphic card. Luminance values corresponding to R-channel outputs of the computer screen were measured with a MINOLTA spectro-photometer. Pairs of red (color coordinates: $\mathrm{x}=.5756$ CIE, $\mathrm{y}=.3679 \mathrm{CIE}$ ) squares or rectangles (see Fig. 1b) were presented to the observers. To vary the relative visibility of the figures of a given pair, the luminance of the background was varied between $3.8,4.8,6.0,8.0$, and $10.4 \mathrm{~cd} / \mathrm{m}^{2}$ in random order within an experimental session. Each stimulus pair contained a brighter figure $\left(11.8 \mathrm{~cd} / \mathrm{m}^{2}\right)$ and a darker figure $\left(2 \mathrm{~cd} / \mathrm{m}^{2}\right)$. The side on which a darker or brighter figure appeared in a pair was varied randomly within and between sessions. Combinations with the different background luminances produced five different levels of contrast or relative visibility for the brighter square/rectangle of a pair, and five different levels of contrast for the darker square/ rectangle. The chromaticity of the red figures was constant.

The contours of all figures were of the same thickness $(1.5 \mathrm{~min}$ of visual arc). The length of each side of a square in the pairs with the positional cue to apparent depth (Fig. 1b, top), was $2.5^{\circ}$ of visual angle. The positional cue systematically favored the square on the right in a given pair. Each of the rectangles in a cross stimulus (Fig. $1 \mathrm{~b}$ middle and bottom) was $3.5^{\circ}$ of visual angle long, and $1.5^{\circ}$ wide. In one condition, the horizontal rectangle of a pair of two rectangles was superimposed on the vertical rectangle ("transparent" crosses; see Fig. 1b, middle) producing local cues of interposition, with the horizontal rectangle appearing to be superimposed on the vertical rectangle. In another condition, the horizontal rectangle of a cross was given a "contour advantage" by producing a stimulus pair with a partially occluded vertical rectangle ("opaque" crosses; see Fig. 1, bottom). Thus, interposition cues and cues of partial occlusion systematically favored the horizontal rectangles in the cross configurations.

\section{Procedure}

The three types of stimulus pairs were presented in separate sessions. Their order of presentation varied randomly. A given stimulus pair was flashed for $128 \mathrm{~ms}$ ( 8 frames) in the center of the computer screen. A small fixation cross, displayed centrally between trials, indicated where to look. Observers had to decide as quickly as possible which figure of the pair seemed nearer than the other by pressing one of two response keys on the computer keyboard. In sessions with pairs of squares, the choice had to be made between the left and the right figure of the pair. In sessions with crosses, observers had to choose between the horizontal and the vertical rectangle. The choice and response time were recorded in each trial. Each of the different stimulus combinations was presented 20 times to each observer in a total of 1,200 trials per observer.

\section{Results and discussion}

The results of Experiment 1 are shown in Figs. 2 and 3. Figures $2 \mathrm{a}$ and $3 \mathrm{a}$ show the probability that a given figure of a pair is perceived as "nearer" than the other as a function of the bright (Fig. 2a) or dark (Fig. 3a) luminance contrast of that figure, and the type of geometric cue presented. Contrast is expressed here in terms of differences between the luminance of a red figure and the luminance of the background. Only probabilities of square figures with positional cues presented on the right-hand side of the screen, and probabilities of horizontal rectangles in the cross configurations with interposition and partial occlusion cues are plotted. Note that the probabilities of squares presented on the left and those of vertical rectangles in the crosses, for a given condition, are obtained by subtracting the probabilities shown in the graphs from 1.

Figure $2 \mathrm{a}$ shows that the probability that a figure appears "nearer" than the other, or probability of "near," increases with increasing luminance contrast of the bright red figures, $F(4,40)=24.423, p<.001$. This result is similar to observations by Dresp et al. (2002) with varying luminance contrast of achromatic stimuli. The increase in probabilities of "near" with increasing relative visibility of bright red figures is particularly strong in the square configurations with the positional cue and in crosses with the interposition cue. The effect

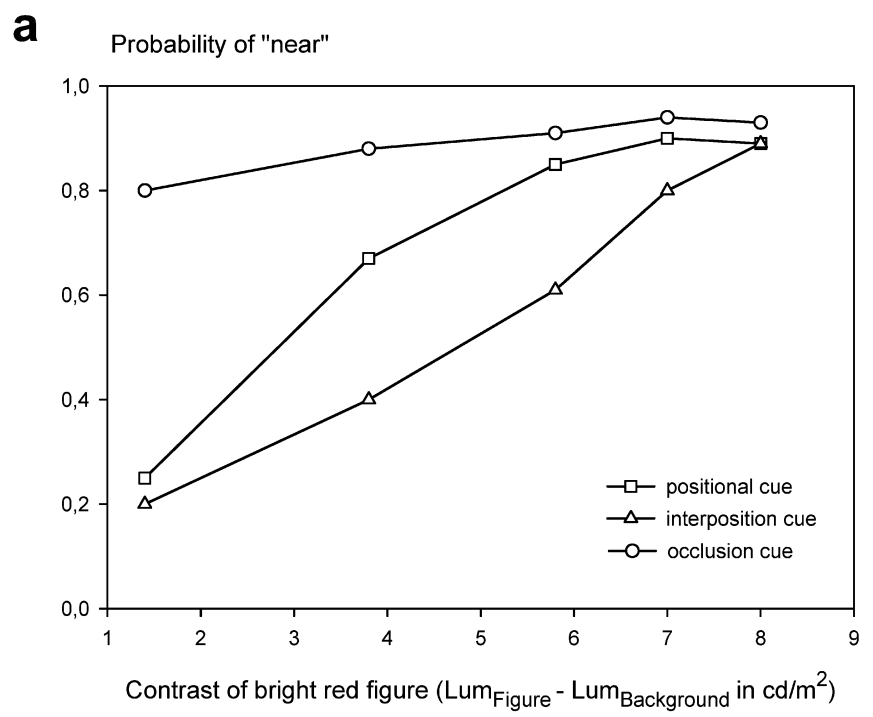

b

Response Time for "near"

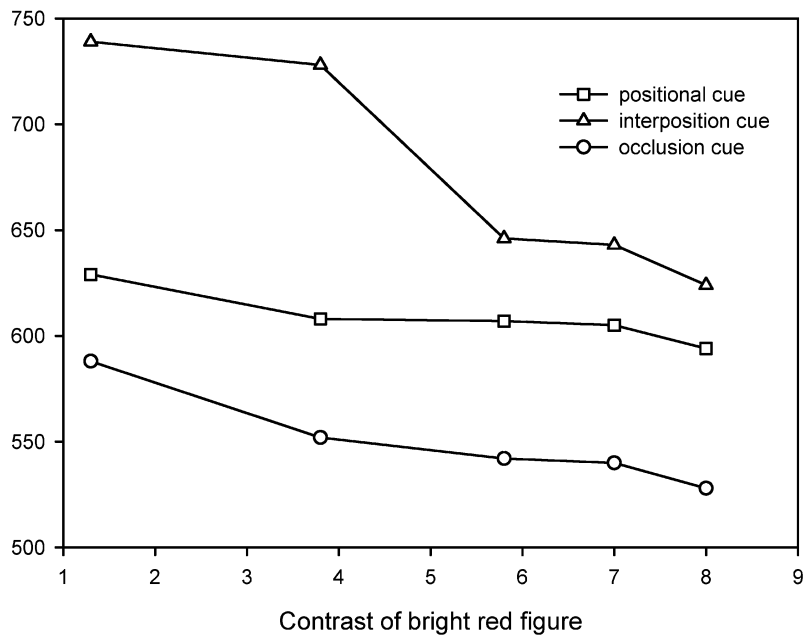

Fig. 2 a The probability that a right-hand side/horizontal figure of a pair is perceived as "nearer" than the other as a function of its luminance contrast and the type of geometric cue presented. The probability that a figure appears "nearer" than the other (probability of "near") increases significantly with increasing luminance contrast of BRIGHT red figures. b Response times for "near" responses to BRIGHT red figures as a function of their luminance contrast and the type of geometric cue presented 
a

Probability of "near"

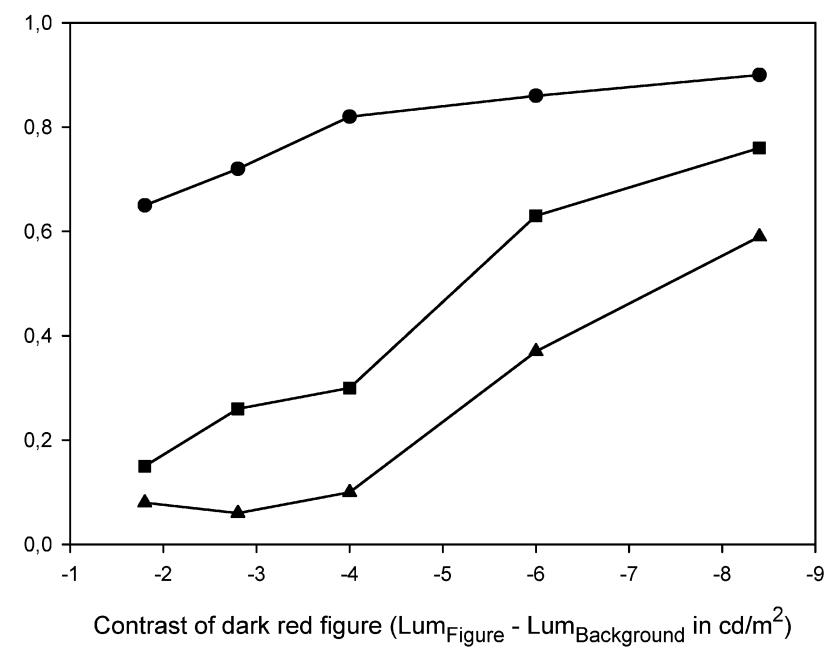

b

Response Time for "near"

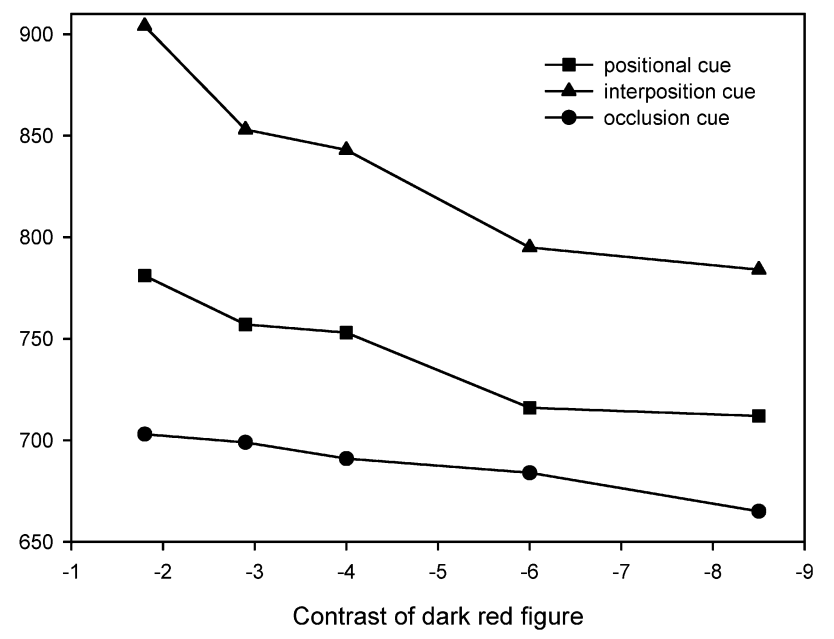

Fig. 3 a The probability that a right-hand side/horizontal figure of a pair is perceived as "nearer" than the other as a function of its luminance contrast and the type of geometric cue presented. The probability that a figure appears "nearer" than the other (probability of "near") increases significantly with increasing luminance contrast of DARK red figures. b Response times for "near" responses to DARK red figures as a function of their luminance contrast and the type of geometric cue presented

of the type of pictorial cue the bright luminance contrasts were combined with is statistically significant, $F(2,20)=54.520, p<.001$. In crosses with the occlusion cue, the horizontal rectangles produce the highest probabilities of "near." Here, the effect of relative visibility is minimal, with probabilities increasing from .8 to .95. Horizontal rectangles supported by the interposition cue produce the lowest probabilities of "near." However, the probabilities increase consistently with the luminance contrast of the stimuli. At the highest contrasts, the differences between probabilities of "near" for figures supported by a positional cue, for figures supported by the interposition cue, and for figures supported by the occlusion cue disappear. In other words, figures with a sufficiently high contrast seem to produce equally strong "near" percepts, whether they are supported by an additional depth cue or not, under the conditions tested here. The interaction between luminance contrast and type of geometric cue presented in the case of bright figures is statistically significant, $F(8,80)=2.40, p<.001$. Figure 3 a shows probabilities of "near" as a function of the contrast of dark red figures, $F(4,40)=22.398, p<.001$. The data are in every respect similar to those found with the bright red figure. The effect of the type of geometric cue the dark luminance contrasts were combined with is statistically significant, $F(2,20)=37.934, p<.001$, and so is the interaction between contrast and type of geometric cue presented in the case of dark figures, $F(8,80)=10.451$, $p<.001$.

The latencies of "near" responses as a function of luminance contrast and type of geometric cue presented are plotted in Figs. $2 \mathrm{~b}$ and $3 \mathrm{~b}$. Figure $2 \mathrm{~b}$ shows that response latencies for "near" decrease systematically as the luminance contrast of a bright red figure increases. The effect of bright luminance contrast on the latencies of "near" responses is statistically significant, $F(4,40)=$ 4.953, $p<.05$. Moreover, geometric cues generating higher probabilities of "near" systematically yield faster responses, with the shortest latencies for the partial occlusion cue, and the longest latencies for the interposition cue. This effect of geometric cues on the latencies of "near" responses to bright red figures is statistically significant, $F(2,20)=9.073, p<.05$, and so is the interaction between luminance contrast and type of geometric cue presented, $F(8,80)=3.972, p<.001$. A comparison between the results shown in Fig. 2 reveals a correlation between the probability of "near" and response latencies, with consistently shorter latencies for higher probabilities.

Similar effects, leading to the same conclusions, are revealed by the results shown in Fig. 3b where response latencies are plotted as a function of the luminance contrast of a dark red figure. The effect of dark luminance contrasts on the latencies of "near responses" is statistically significant, $F(4,40)=3.704, p<.05$, and so is the effect of geometric cues, $F(2,20)=3.904, p<.05$. As expected, the interaction between luminance contrast and geometric cues is also statistically significant, $F(8,80)=5.457, p<.001$. A comparison of Fig. 3a and $b$ reveals, as in the case of the bright red figures, a clear correlation between probabilities of "near" and the corresponding response latencies, with consistently shorter latencies for higher probabilities.

Apart from the observation that "near" judgments were, on average, shorter for bright red figures than for dark red figures, there were no systematic differences in probabilities of "near," or response latencies for "near," between conditions where a bright red figure overlapped a dark one, and conditions where a dark red figure overlapped a bright one. The fact that average response times for bright colors are shorter than average response times for dark colors is similar to Dresp et al.'s (2002) 
observation with achromatic stimuli, where bright figures produced shorter mean response latencies than dark figures. These findings are likely to be explained by stronger probability summation of luminance intensities with positive contrast polarity at early stages of visual contrast integration (e.g., Tolhurst, 1975).

\section{Experiment 2: Color contrast versus partial occlusion, interposition, and relative height in the visual field}

Having ruled out any major functional asymmetry in the perception of "near" from colored figures with dark and bright luminance contrasts, we investigated the effects of wavelength, or color contrast, of isoluminant red, green, and white stimuli on the probability that a given figure in a pair is perceived as "nearer" than the other. Backgrounds with dark and light luminance were used to bring to the fore the extent to which the effect of mere color contrast on "near" percepts interacts with background luminance, as shown by previous findings from experiments on color stereopsis (e.g., Dengler \& Nitschke, 1993). Interactions of color contrast with geometric cues of partial occlusion, interposition, and relative height in the visual field were examined. As in Experiment 1, we designed the figure pairs to produce cues of relative height that systematically favored squares displayed on the right. Interposition cues and partial occlusion cues systematically favored the horizontal rectangles in the cross configurations. Again, such a bias would produce a probability of 1 for squares on the right and horizontal rectangles to be perceived as "nearer" if color, or wavelength, had no effect on the depth judgments.

\section{Method}

Participants

The 11 participants from Experiment 1 took part in Experiment 2.

Stimuli

The stimuli were presented using the same equipment as in Experiment 1. Pairs of red (color coordinates: $\mathrm{x}=.5765$ CIE, $\mathrm{y}=$ .3679 CIE) and green $(\mathrm{x}=.2863 \mathrm{CIE}, \mathrm{y}=.3679 \mathrm{CIE})$ or red $(\mathrm{x}=$ .5765 CIE, $\mathrm{y}=.3679$ CIE $)$ and white $(\mathrm{x}=.1863$ CIE, $\mathrm{y}=.3679$ CIE) squares or rectangles of physical isoluminance $\left(12 \mathrm{~cd} / \mathrm{m}^{2}\right)$ were displayed. A measurement of $12 \mathrm{~cd} / \mathrm{m}^{2}$ was found to be close enough to individual points of psychophysical isoluminance for the three colors. Psychophysical isoluminance had been assessed beforehand by two separate procedures consisting of a classic flicker test on the one hand and of minimally distinct border adjustments on the other, as in Dresp and Fischer (2001). In the present experimental display, psychophysical isoluminance varied between $11.82 \mathrm{~cd} / \mathrm{m}^{2}$ and $12.54 \mathrm{~cd} / \mathrm{m}^{2}$ for green, and between $12.22 \mathrm{~cd} / \mathrm{m}^{2}$ and $13.01 \mathrm{~cd} / \mathrm{m}^{2}$ for red when testing for green $/ \mathrm{red}$ isoluminance. Variations between $11.72 \mathrm{~cd} / \mathrm{m}^{2}$ and $12.23 \mathrm{~cd} / \mathrm{m} 2$ for white and between $12.03 \mathrm{~cd} / \mathrm{m}^{2}$ and $12.89 \mathrm{~cd} / \mathrm{m}^{2}$ for red were found when white/red isoluminance was assessed. The luminance of the background was $.01 \mathrm{~cd} / \mathrm{m}^{2}$ (black) in one set of conditions and
$10 \mathrm{~cd} / \mathrm{m}^{2}$ (light gray) in another. Dark and light backgrounds were presented in a random order within an experimental session. Each stimulus pair contained two isoluminant figures of a different color. In one set of conditions, a red figure was presented together with a green figure; in another set of conditions, a red figure was presented together with a white figure. The side on which a given color appeared in a pair was varied randomly. Red/white stimulus pairs and $\mathrm{red} /$ green stimulus pairs were presented in separate sessions. The order of presentation of the sessions varied randomly. As in the previous experiment, the contours of all figures were of equal width (1.5 min of visual arc). The length of each side of a square in the control pair, which did not contain any specific feature cues to apparent depth, was $2.5^{\circ}$ of visual angle. Each of the rectangles in a cross stimulus was $3.5^{\circ}$ of visual angle long, and $1.5^{\circ}$ wide.

\section{Procedure}

A given stimulus pair was flashed for $128 \mathrm{~ms}$ ( 8 frames) in the center of the computer screen. A small fixation cross, displayed centrally between trials, indicated where to look. Observers had to decide as quickly as possible which figure of the pair seemed nearer than the other by pressing one of two response keys on the computer keyboard. In sessions with pairs of squares, the choice had to be made between the left and the right figure of the pair. In sessions with crosses, observers were asked to choose between the horizontal and the vertical rectangle. The choice and response time were recorded in each trial. Each of the different stimulus combinations was presented 20 times to each observer in a total of 680 trials per observer.

\section{Results and discussion}

The results of Experiment 2 are shown in Fig. 4. In Fig. $4 \mathrm{a}$, the probability that a given figure of a pair is perceived as "nearer" than the other is plotted as a function of the color of that figure, the brightness of the background, and the type of geometric cue presented. Only probabilities of square figures with positional cues presented on the right-hand side of the screen, and probabilities of horizontal rectangles in the cross configurations with interposition and partial occlusion cues are plotted. Note that the probabilities of squares presented on the left and those of vertical rectangles in the crosses for a given condition are obtained by subtracting the probabilities shown in the graphs from 1.

The data reveal that red rectangles supported by occlusion cues produce the highest probabilities of "near." These probabilities are close to 1 , and do not vary much with the brightness of the background on which the figures were presented. White rectangles supported by interposition cues are found to be hardly ever perceived as "nearer" than their red vertical counterparts. The effect of the type of geometric cue presented is statistically significant, $F(2,20)=37.300, p<.001$. Red figures generally produce higher probabilities of "near" compared with green or white figures. For example, red squares presented on the right in pairs of stimuli with a positional cue to "near" produce noticeably higher probabilities of "near" than right-hand side white squares or right-hand side green squares. The same holds true for horizontal red rectangles supported by cues of interposition or partial occlusion when compared with the green or the white 


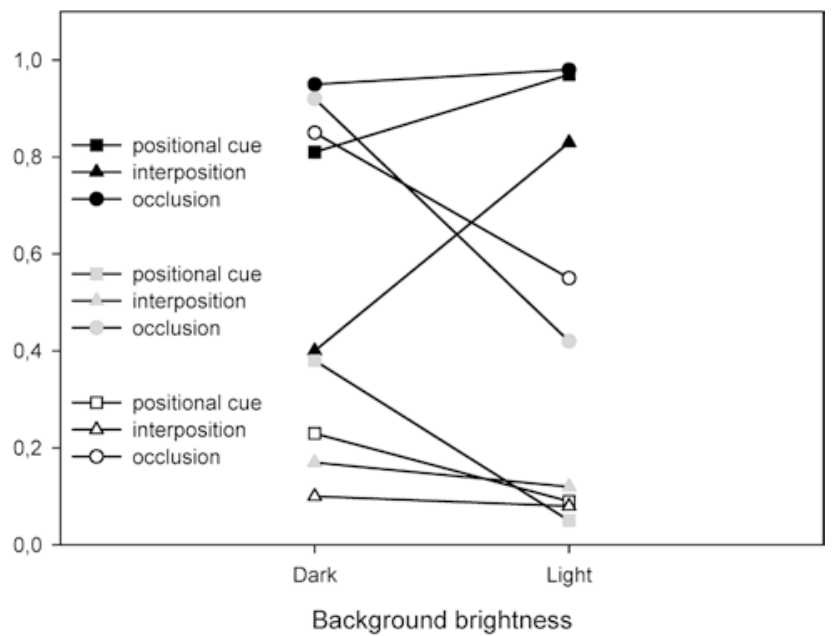

b

Response Time for "near"

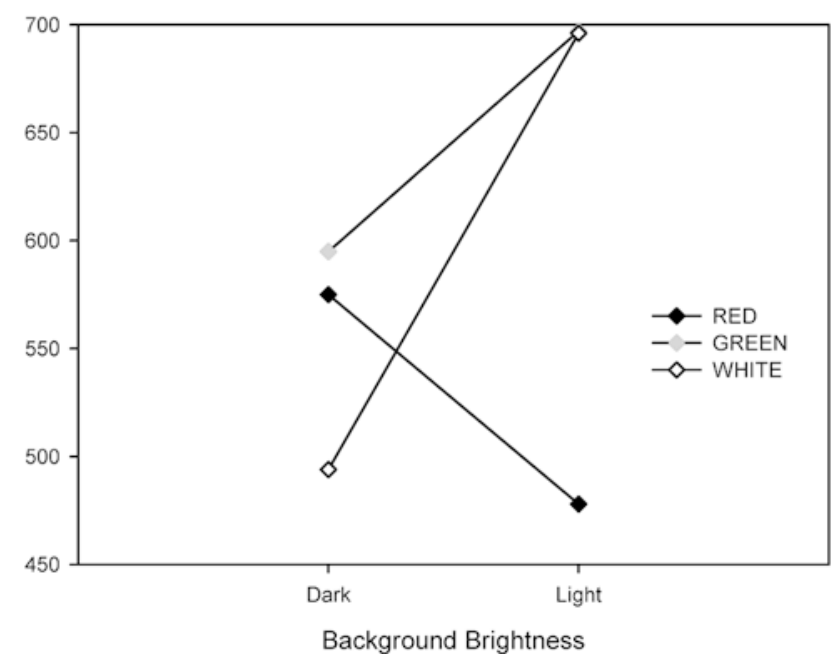

Fig. 4 a The probability that a right-hand side/horizontal figure of a stimulus pair is perceived as "nearer" than the other as a function of the color of that figure, the brightness of the background, and the type of additional cue presented. Black symbols stand for red figures, gray symbols stand for isoluminant green figures, and white symbols stand for isoluminant white figures. The data bring to the fore complex interactions between color and the type of geometric cue the color is combined with. The effect of these cue combinations varies with the brightness of the background on which they are presented. b Response times for "near" judgments as a function of the color of the figure seen as nearer and the brightness of the background on which the figure pair was presented

ones (see Fig. 3). The effect of color on the probability of "near" is statistically significant when comparing red with green, $F(1,10)=46.531, p<.001$, and when comparing red with white, $F(1,10)=73.253, p<.001$. We observe an interaction between the effect of color and the effect of background brightness. Red figures are more likely to be perceived as "nearer" when presented on a light background, while white and green figures are more likely to be perceived as "nearer" when presented on a dark background. The interaction between the effect of color and the effect of background brightness is statistically significant in the case of red/white pairs, $F(1,10)=$ $17.919, p<.002$, and in the case of red/green pairs, $F(1,10)=34.966, p<.001$. Interactions between the effect of color and the effect of the type of geometric cue with which a color is combined are also statistically significant in the case of red/white pairs, $F(2,20)=16.469$, $p<.001$, as well as for red/green pairs, $F(2,20)=14.841$, $p<.001$. Red rectangles supported by occlusion cues produce the highest probabilities of "near," white rectangles supported by interposition cues the lowest. Red figures with a positional cue produce noticeably higher probabilities of "near" than white or green figures combined with interposition cues or cues of partial occlusion.

In Fig. 4b, response times for "near" responses are plotted as a function of the color of the figure seen nearer, and the brightness of the background on which the figure was presented. The response latencies are found to correlate with probabilities of "near" shown in Fig. 4a, with systematically shorter latencies for colors yielding higher probabilities of "near." The interaction between the effect of color and the effect of background brightness on response latencies is statistically significant, $F(2,20)=5.547, p<.05$. The effect of color or of geometric cues as such on response latencies is not statistically significant, but the interaction between color and geometric cues is, $F(4,40)=2.950, p<.05$. The shortest response latencies were found with red supported by the positional cue on the light background (444 ms), white supported by the partial occlusion cue on the dark background (448 ms), red supported by the partial occlusion cue on the light background (477 ms), and white supported by the positional cue on the dark background (486 ms). The longest response latencies were found with green supported by the interposition cue on a light background (712 ms), green supported by the positional cue on a light background $(705 \mathrm{~ms})$, white supported by the interposition cue on the light background $(702 \mathrm{~ms})$, and white supported by the positional cue on the light background (692 ms).

\section{General discussion}

The results of the present study reveal the complexity of interactions between color and geometric cues in the perception of apparent depth. They allow us to identify and to unravel some of the major causal factors underlying this complexity. The findings from Experiment 1 clarify issues regarding a possible functional asymmetry between bright and dark contrasts (e.g., Magnussen \& Glad, 1975; Dengler \& Nitschke, 1993) by showing that a basic functional asymmetry between luminance contrasts of dark and light colors, which may influence the process generating depth percepts, can be ruled out. Geometric cues of partial occlusion and interposition were found to compete with strong luminance contrasts of the color red in the same way as they had previously been found to 
compete with strong achromatic contrasts (Dresp et al., 2002), with no major difference between dark and bright contrasts. This observation confirms the fundamental working hypothesis of Grossberg's FACADE theory (Grossberg, 1997) that luminance contrasts of achromatic stimuli and luminance contrasts of colors use the same mechanism to produce percepts of apparent depth.

The findings from Experiment 2 show that color contrast alone is not an independent depth cue, as is widely assumed, and that the effect of a given color on perceived depth in pictorial displays depends on the type of geometric cue the color is combined with. Moreover, the ways in which color interacts with geometric cues to depth is found to depend on whether the isoluminant configurations are seen on a light or a dark background. This observation suggests that depth percepts in colored stimuli are strongly influenced by non-linearities due to changes in illumination levels, or so-called visual adaptation levels. While an interaction between color and adaptation levels is consistent with the classic explanation of pictorial depth from color in terms of prismatic effects in human optics (see also Simonet \& Campbell, 1990), commonly referred to as color stereopsis, the triple interaction between color, illumination level, and geometric constraints is definitely not. We therefore conclude that color stereopsis cannot be the sole determinant of pictorial depth from color, as is often quite readily assumed (e.g., Dengler \& Nitschke, 1993).

The response latency of "near" judgments, in both experiments, correlates with the probability of "near," with higher probabilities of "near," systematically producing shorter latencies. This major finding strongly suggests that selective visual attention reliably detects the most likely depth cue combination in a configuration. This is consistently reflected by a faster selection of more likely cue combinations. Our results have major implications for several important theoretical issues.

\section{Implications for FACADE theory}

Our findings are largely consistent with the FACADE model of Grossberg (1997), which proposes a two-stage model of visual mechanisms to explain how twodimensional pictures give rise to three-dimensional perceptions of "near" and "far." The model emphasizes the role of geometric and contrast properties of visual stimuli, which interact to generate a depth percept. Like Dresp et al.'s (2002) findings with achromatic stimuli, the present findings with colored stimuli confirm the FACADE prediction that a geometric cue of partial occlusion cues wins against an interposition cue in competition with a strong luminance cue. This is explained by the relative boundary advantage produced by the partial occlusion cue compared with the interposition cue (see again Fig. 1a). This relative boundary advantage generates comparatively stronger signals at the first processing stage of the FACADE model, and thus the partial occlusion cue will stand a better chance than the interposition cue of "surviving" competition with other cues at further stages of processing.

In addition, the present data add a new finding that further clarifies the status of geometric cues as dealt with by the FACADE model. Both Experiment 1 and Experiment 2 clearly show that the cue to relative height in the visual field, or positional cue, produces higher probabilities of "near" and faster perceptual decisions than the interposition cue, but lower probabilities and slower perceptual decisions than the partial occlusion cue. In configurations with the positional cue, no geometric constraint other than that of spatial location influences the process that leads to perceived depth. According to grouping rules promoted by the FACADE model, the positional cue should not have a geometric advantage over either of the two other cues. In our study, the positional cue is found to win over the interposition cue, and is found to lose out against the partial occlusion cue. This interesting and new finding is possibly explained by a strong influence of spatial location on visual attention. The FACADE theory predicts that expectation triggered by cues of spatial location is a particularly powerful attractor of visual attention.

Moreover, the effect of the positional cue compared with that of additional geometric properties such as partial occlusion or interposition indicates that several cues combined in a given configuration do not produce simple summation effects. Such a conclusion is consistent with a cue-mixture model where different probabilistic weights are given to different depth cues, and where the weight of a given cue is conditional upon the relative weight of other cues (Knill, 2003).

Implications for probabilistic models of depth-cue combination

Knill's (2003) cue mixture model is, like the FACADE theory, based on cue cooperation. Cue competition is, as in the FACADE theory, the inevitable consequence of cue selection. Although all cues available in a given configuration cooperate in the first instance, cues with little relative weight may subsequently be overridden by a cue with stronger relative weight. However, unlike the FACADE theory, the cue mixture model does not attempt to link visual cortical function to mechanisms of depth perception. It uses Bayesian principles to compute conditional probability functions, which are the product of the likelihood functions for each given cue. A conditional probability function predicts when a single cue will have enough weight to effectively determine the resulting percept. Depth perception is thus seen as a probabilistic process where the most likely cue, or cue combination, is selected. The response latencies from the present experiments show that the most likely depth cue combinations systematically produce the fastest perceptual judgments, which is generally consistent with the idea of depth perception as a probabilistic process. 
Nevertheless, there has to be a mechanism in the human brain that drives this probabilistic process. The FACADE theory proposes selective visual attention as a candidate, referring to earlier results by Nakayama and Silverman (1986), which have shown that combinations of depth and color are detected faster in visual search than color or depth alone because the conjunction of both attributes facilitates attentional selection. The correlation between probabilities of "near" and response latencies we find in our present results are consistent with such an interpretation.

The status of color as depth cue

One aspect of the findings of Experiment 2 is that the effect of a given color on depth percepts may depend considerably on whether the stimulus is presented on a dark or a light background. In our experiments, the isoluminant configurations presented on the light background had a lower luminance contrast than the isoluminant configurations presented on the dark background, yet the luminance contrast of both figures in a given pair was always identical in a given configuration. Thus, differences in illumination level can only account for the fact that a given color produced different effects on different backgrounds. This result is consistent with earlier observations by Simonet and Campbell (1990) on the effect of illumination levels in color stereopsis. It reflects the contribution of color stereopsis to depth perception in two-dimensional stimuli at early stages of visual processing (e.g., Dengler \& Nitschke, 1993); however, color stereopsis alone cannot explain all of our findings from Experiment 2, which show that the effect of color contrast interacts with the type of geometric cue the color is combined with. For example, we find that partial occlusion cues combined with the colors green or white produce weaker depth percepts and slower perceptual judgments than partial occlusion cues combined with the color red, regardless of the background intensity. At the same time, we find that the presumably weaker interposition cue combined with the color red produces stronger depth percepts and faster perceptual judgments than partial occlusion cues combined with the colors green or white. This is an important finding because it shows that a color cue, here red, can override the boundary advantage in the configurations with partial occlusion. This effect is not predicted by the FACADE model or any theory we know of. It seems to highlight the presumed particular status of the color red as a cue to depth (e.g., Bugelski, 1967).

\section{When does "red" mean "near"?}

Red stimuli are not, however, inevitably seen as "nearer" in the visual field than stimuli with other colors. Our results from Experiment 2 show that the highest probabilities of "near" and the fastest perceptual judgments are produced by red figures combined with a partial occlusion cue or a positional cue presented on a light background. This suggests that the color red is, indeed, a potentially strong candidate as a depth cue with, initially, a strong relative weight. However, white figures combined with a partial occlusion cue produce stronger depth percepts and faster perceptual judgments than red figures combined with an interposition cue presented on a dark background. This result clarifies that, despite a given advantage due to wavelength and color stereopsis, the color red does not always benefit from this advantage, nor does it automatically attract visual attention away from other stimuli in the visual field. It may only do so when certain geometric constraints are met.

In conclusion, the results of Experiment 2 suggest that cortical mechanisms contribute to depth perception from color. Grossberg's FACADE model (Grossberg, 1997) explains how cortical mechanisms generate perceived depth in two-dimensional geometric configurations. The hypothesis of a cortical contribution to depth perception from color does clearly not receive enough consideration, despite the fact that it is highly consistent with current neurophysiological data (e.g., Desimone \& Schein, 1987).

\section{References}

Brewster, D. (1851) Notice of a chromatic stereoscope. Philosophical Magazine, 4th Series, 3, 31.

Bruno, N., \& Cutting, J. E. (1988) Minimodularity and the perception of layout. Journal of Experimental Psychology: General, 117, 161-170.

Bugelski, B. R. (1967) Traffic signals and depth perception. Science, 157, 1464-1465.

Da Vinci, Leonardo (1651) Trattato della Pittura di Leonardo da Vinci. Scritta da Raffaelle du Fresne. Paris: Langlois.

Dengler, M., \& Nitschke, W. (1993) Color stereopsis: a model for depth reversals based on border contrast. Perception \& Psychophysics, 53, 150-156.

Desimone, R., \& Schein, S. J. (1987) Visual properties of neurons in area V4 of the macaque: sensitivity to stimulus form. Journal of Neurophysiology, 57, 835-868.

Dresp, B., \& Fischer, S. (2001) Asymmetrical contrast effects induced by luminance and color configurations. Perception \& Psychophysics, 63, 1262-1270.

Dresp, B., \& Grossberg, S. (1999) Spatial facilitation by color and luminance edges: Boundary, surface, and attentional factors. Vision Research, 37, 913-924.

Dresp, B., Durand, S., \& Grossberg, S. (2002) Depth perception from pairs of overlapping cues in pictorial displays. Spatial Vision, 15, 255-276.

Egusa, H. (1983) Effects of brightness, hue, and saturation on perceived depth between adjacent regions in the visual field. Perception, 12, 167-175.

Gibson, J. J. (1950) Perception of the visual world. Boston, MA: Houghton-Mifflin.

Goolsby, B. A., \& Suzuki, S. (2001) Understanding priming of color-singleton search: roles of attention at encoding and "retrieval". Perception \& Psychophysics, 63, 929-944.

Grossberg, S. (1997) Cortical dynamics of three-dimensional figureground perception of two-dimensional figures. Psychological Review, 104, 618-658.

Hartridge, H. (1947) The visual perception of fine detail. Philosophical Transactions of the Royal Society, 232, 519-671. 
Knill, D. (2003) Mixture models and the probabilistic structure of depth cues. Vision Research, 43, 831-854.

Landy, M. S., Maloney, L. T., \& Young, M. (1995) Measurement and modeling of depth cue combination: In defense of weak fusion. Vision Research, 35, 389-412.

Magnussen, S., \& Glad, A. (1975) Brightness and darkness enhancement during flicker: Perceptual correlates of neuronal Band D- systems in human vision. Experimental Brain Research, 22, 399-413.

Nakayama, K., \& Silverman, G. H. (1986) Serial and parallel processing of visual feature conjunctions. Nature, 320, 264-265.

Nakayama, K., Shimojo, S, \& Ramachandran, V. S. (1990) Transparency, relation to depth, subjective contours, luminance, and neon color spreading. Perception, 19, 497-513.

O'Shea, R. P., Blackburn, S. G., \& Ono, H. (1994) Contrast as a depth cue. Vision Research, 34, 1595-1604.

Rock, I., Shallo, J., \& Schwartz, F. (1978) Pictorial depth and related constancy effects as a function of recognition. Perception, 7, 3-19.
Rohaly, A. M., \& Wilson, H. R. (1993) The role of contrast in depth perception. Investigative Ophthalmology and Visual Science, 34, 1437.

Ross, H. E. (1967) Water, fog, and the size distance invariance hypothesis. British Journal of Psychology, 58, 301-313.

Simonet, P., \& Campbell, M. C. W. (1990) Effect of luminance on the directions of chromatostereopsis and transverse chromatic aberration observed with natural pupils. Ophthalmic \& Physiological Optics, 10, 271-279.

Tolhurst, D. J. (1975) Reaction times in the detection of gratings by human observers: A probabilistic mechanism. Vision Research, $15,1143-1149$.

Verhoeff, F. H. (1928) An optical illusion due to chromatic aberration. American Journal of Ophthalmology, 11, 898-900.

Yantis, S., \& Jones, E. (1991) Mechanisms of attentional selection: Temporally modulated priority tags. Perception \& Psychophysics, 50, 166-178 